\title{
WORKING PARTIES
}

\section{WP03}

Stem cell therapy in type 1 diabetes. how do we convince the diabetes community?

M. C. Oliveira Rodrigues ${ }^{1, *}$

${ }^{1}$ Ribeirão Preto Medical School, University of São Paulo, Ribeiro Preto, Brazil

Abstract: Type 1 diabetes is an immune-mediated disorder that affects insulin-producing pancreatic beta cells. Destruction of beta cells progressively decreases endogenous insulin secretion. The disease remains subclinical for the initial months to years, but when insulin production achieves a critical level and glucose homeostasis is no longer possible, diabetes becomes clinically evident. Itis long known that at diagnosis, patients still preserve approximately 15 to $20 \%$ of their original beta cell mass. Attempts to preserve this residual pancreatic function have been described for the past 20 years. Numerous immunosuppressive and immunomodulating drugs have been investigated both prophylactically and at diagnosis. While several placebo-controlled trials have successfully shown a lesser degree of beta cell destruction in immunosuppressed patients, none were able to effectively increase pancreatic function or to substantially decrease exogenous insulin injections. In the past 10 years, autologous hematopoietic stem cell transplantation (AHSCT) has been investigated as a possible immunoablative approach to cease beta cell destruction early after diagnosis of type 1 diabetes. Studies have demonstrated that AHSCT was able to increase endogenous pancreatic function, measured through C-peptide levels, for at least 48 months (4 years) after transplantation. Furthermore, most patients remained insulin-free, transiently or continuously, for a median duration of 44 (range $6-132$ ) months after AHSCT. Although improvements in the protocol are still required to increase duration of the transplantmediated immunological effects, AHSCT is so far the most effective intervention to improve pancreatic function and glycemic control of type 1 diabetes patients.

Disclosure of Interest: None declared.

\section{WP10}

Molecular pathogenesis of adult acute lymphoblastic leukemia

S. Izraeli, , ,*

${ }^{1}$ Functional Genomics Cancer Research Center, Sheba Medical Center, Ramat Gan, ${ }^{2}$ Human Molecular Genetics and Biochemistry, Tel Aviv University, Tel Aviv, Israel

Abstract: Acute lymphoblastic leukemia (ALL) is a heterogeneous disease characterized by several genetic subgroups. The good prognosis subgroups (ETV6-RUNX1 translocation and high hyprediploid ALLs) are common in children but rare in adults. The talk will focus on recent discoveries of molecular subtypes of ALL that are relevant to adults and may be amenable to targeted therapies. The first group is kinase driven ALLs, more commonly known as "Philadelphia like" or "BCR-ABL like" ALLs. This group can be generally divided into two large subgroups. One that is driven by $A B L$ signaling pathway and should therefore be sensitive to $A B L$ inhibitors, and the other that is driven by the JAK-STAT pathway. The relevance of commercially available JAK inhibitors for treatment of these JAK-STAT leukemias is unknown. The precise diagnostic approach for the identification of kinase driven leukemias is undecided yet. Importantly a significant fraction to T-ALLs that is dependent on IL7 signaling may also be sensitive to JAK inhibitors. Another therapeutically relevant discovery is the distinction between early pre-B ALLs and ALLs that depend on signaling from the pre-B cell receptors (pre$B C R$ ). The second group, whose frequency in adults is currently unclear, is characterized by high expression of BCL6 and by sensitivity to Dasatinib and possibly also to other inhibitors of $B C R$. Thus new discoveries of molecular subtypes of ALL are likely to expand the role of kinase inhibitors in the treatment of ALL.

Disclosure of Interest: None declared.

\section{WP11}

TBI-based conditioning for ALL - can we improve? S. Giebel ${ }^{1, *}$ on behalf of Acute Leukemia Working Party ${ }^{1}$ Bone Marrow Transplantation and Onco-Hematology, Maria Sklodowska-Curie Memorial Cancer Center and Institute of Oncology, Gliwice Branch, Gliwice, Poland

Abstract: Total body irradiation (TBI) is widely used as part of conditioning prior to hematopoietic stem cell transplantation. Results of retrospective, registry-based studies, indicate that for patients with acute lymphoblastic leukemia (ALL), TBI-based treatment is associated with significantly reduced risk of relapse and improved leukemia-free survival compared to regimens based on chemotherapy. Unfortunately, when used in myeloablative doses, TBI is associated with significant morbidity, including late effects such as endocrine deficiencies, pulmonary complications or secondary malignancies. Therefore attempts are needed to reduce the toxicity while preserving or increasing the efficacy of TBI. The potential way to improve outcome is to optimize TBI techniques with regard to dose homogeneity, dosimetry, methods of immobilization and organ shielding. As demonstrated by recent EBMT survey, very high heterogeneity of these techniques is observed across countries and centers in Europe. As well, the total doses, ways of fractionation and dose rates vary, indicating the need for standardization. Another area of investigation is combining TBI with chemotherapy, beyond traditional use of cyclophosphamide. Finally, there are attempts to target irradiation using more selective techniques such as total marrow irradiation or total lymphoid irradiation, which may be performed using either helical tomotherapy or volumetric modulated arc therapy.

Disclosure of Interest: None declared.

\section{WP13}

Allogeneic stem cell transplantation (Allo-SCT) for Acute Lymphoblastic Leukemia (ALL) in adult patients - are we doing better?

A. Nagler ${ }^{1, *}$ on behalf of ALWP

${ }^{1}$ Division of Hematology, Chaim Sheba Medical Center Tel Has homer, Israel

Abstract: Acute lymphoblastic leukemia (ALL) is a heterogenic neoplasm of the lymphocyte precursor cells. Among adult patients (pts) it is a relatively rare malignancy with an historical curability rate of around $30 \%$ at 5 years. Allo-SCT is the treatment of choice for adult pts with ALL after first relapse, 
and is also recommended for high-risk pts in first CR. Recent developments including minimal residual disease (MRD) evaluation and monitoring is emerging as the most important prognostic factor in determining which of the pts, especially those with standard risk, should be referred to allo-SCT. Tyrosine kinase inhibitor (TKI) therapy allows a much higher proportion of ph+ ALL pts to attain remission and proceed to allo-SCT with improved results. Post-transplant TKI maintenance therapy may also provide survival benefit. Reducedintensity conditioning (RIC) regimens and the availability of unrelated and recently alternative donors for allo-SCT, are reasonable options for pts who would otherwise be ineligible for transplant due to age, co-morbidities, or lack of an HLA matched sibling donor. Novel compounds like the Bi-specific T-cell engagers (BiTEs) antibody-Blinatumomab and the chimeric antigen receptor (CAR) CD19 $\mathrm{T}$ cells pre-or post allo-SCT may further improve results. Previous transplantation studies mainly for AML and MDS indicated improvement of allo-SCT outcome in recent years due to better supportive care, high resolution HLA typing and anti-fungal and viral prophylaxis, resulting in about $50 \%$ in transplant related mortality. However, paucity of data is available for allo-SCT in ALL. We therefore recently analyzed if results of allo-SCT from unrelated (UD) and Haploidentical donors (HD) improved over time and tried to identify prognostic factors using the data registered with the ALWP of the EBMT. Specifically, we analyzed 2178 adult pts with ALL that underwent allo-SCT from UD in the various EBMT centers and reported to the ALWP between 1993 to 2012. Transplantation outcome for 3 subsequent periods: $1993-2002$ vs $2003-2007$ vs $2008-2012$, respectively in various age groups were compared. Our study indicate that outcome of allo-SCT from UD for adults with ALL in CR improved significantly over time with an overall survival rate reaching $65 \%$. The improvement was mainly related to reduced relapse risk and was particularly prominent for 36-55 years old pts and those with $\mathrm{Ph}+$ disease. As for allo-SCT from $\mathrm{HD}$, in order to investigate the outcome of unmanipulated haploidentical SCT (haplo) for ALL we performed a retrospective analysis of 264 adult pts transplanted in EBMT centers from 2007 to 2014. The study results indicated that unmanipulated haplo-SCT seems to be an effective mode of therapy for adults pts with ALL with 49\% OS and 45\% LFS at 2 years post transplantation in pts in first CR and impressive 39\% GRFS. Disease status at transplant and use of PTCy were the main factors affecting outcomes. In 2 additional recent studies we analyzed Thiotepa based allo-SCT for adults with ALL and compared non T depleted Haplo-SCT to unrelated transplants in adult pts with ALL not in remission. Overall we may conclude that "We are doing better" thanks to improve in transplantation strategies, donor availability, novel compounds and conditioning, pre-and post-transplantation, targeted therapies as well as MRD monitoring and the availability of TKls for ph+ ALL. Future means to decrease transplant related toxicity and relapse are in need in order to further improve outcomes.

Disclosure of Interest: None declared.

\section{WP14}

PH+ ALL-not any more a devastating disease?

E. Brissot ${ }^{1, *}$ on behalf of ACUTE LEUKEMIA WORKING PARTY

${ }^{1}$ Hematologie, Service d'Hématologie Clinique et de Thérapie Cellulaire, Hôpital Saint Antoine, APHP, Paris, France

Abstract: Philadelphia chromosome-positive acute lymphoblastic leukemia (Ph+ ALL) is characterized by expression of oncogenic fusion product BCR-ABL1, resulting from reciprocal translocation between chromosomes 9 and 22 [t(9;22)(q34; q11.2)]. Historically, under the sole chemotherapy, it had a very poor prognosis, with a median survival of 8 months. The incorporation of BCR-ABL1 tyrosine kinase inhibitors (TKIs) into chemotherapy has significantly improved its outcomes. The best results have been obtained with immediate TKIs incorporation at $\mathrm{Ph}+\mathrm{ALL}$ recognition and with prolonged and continuous exposure of TKIs. Allogeneic stem cell transplantation (HSCT), either in the pre-TKI or post-TKI era, offers significant improvement in OS and is recognized as the mainstay of $\mathrm{Ph}+\mathrm{ALL}$ treatment. With TKIs, higher remission rates were achieved, thus allowing more patients to proceed with HSCT. Some studies reported the lack of survival benefit with imatinib addition to chemotherapy in the absence of HSCT. However, the indispensability of HSCT was scrutinized following data from pediatric Ph+ ALL patients. Moreover, second-generation TKIs, as dasatinib that inhibits SRC kinases, are more potent than imatinib. Ravandi et al reported that patients receiving dasatinib with induction therapy chemotherapy, continuously throughout consolidation and then during maintenance therapy, obtained excellent CR and cytogenetic response, while in CR1 patients, no outcome differences were observed in patients whether or not they have undergone HSCT. Recently, in the GRAAPH-2005 study, the 3-year survival was similar between patients who underwent a HSCT and those who received an autograft. Finally, the predictive value of minimal residual disease by real-time quantitative polymerase chain reaction and by multiparameter flow cytometry may identify different prognostic groups in CR1 patients, for whom the best consolidation strategies remain to be validated. All these very recent advances have opened the debate: should all Ph+ALL patients be considered to have a devastating disease?

Disclosure of Interest: None declared.

\section{WP15}

Treatment of Acute Leukemias by CAR-engineered CIK cells through an improved non viral platform

E. Biagi $i^{1, *}$, C. F. Magnani ${ }^{1}$, S. Tettamanti ${ }^{1}$, A. Biondi ${ }^{1}$

${ }^{1}$ Pediatrics, San Gerardo Hospital, University of Milano Bicocca, Centro di Ricerca "Matilde Tettamanti", Monza, Italy

Abstract: Adoptive immunotherapy by effector $T$ cell gene-manipulation with chimeric antigen receptors (CARs) is an emerging therapeutic option in relapsed cancer, such as hematological malignancies. However, the clinical application of CAR-engineered cells relies on solving critical issues concerning the profile of efficacy, safety and feasibility of cell manufacturing and gene therapy. In order to rescue the concerns associated to viral vectors, we have explored the use of the Sleeping Beauty Transposon (SB)-mediated gene transfer to establish a novel GMP-grade platform by using cytokine-induced killer (CIK) cells. We generated and propagated $\mathrm{CAR}^{+}$CIK cells with the purpose of optimizing cell expansion and, at the same time, minimizing cell manipulation and specific selection. We obtained around $60 \%$ stable expression of two distinct 3rd generation CARs, specific for the acute myelogenous leukemia (AML) $\mathrm{CD}_{123^{+}}$or acute lymphoblastic leukemia (ALL) $\mathrm{CD} 19^{+}$blasts. The procedure minimally affected the phenotype of CIK cells and was effective in inducing $T$ cell expansion, with a fold increase suitable for future translation into adoptive cell therapy clinical protocols. Modified CIK cells displayed stable expression of CD123.CAR or CD19.CAR, persistence of a subset with memory phenotype and efficient lysis of leukemic cell lines and primary blasts. Specific target recognition by the CAR elicited cytokine secretion and cell proliferation. Adoptive transfer of CD123. CAR or CD19.CAR CIK cells exhibited control of AML and ALL in mice, respectively. Notably, no evidence of integration enrichment near cancer-related genes neither presence of transposase enzyme at the end of differentiation was detected, assuring the safety profile of SB system. This easy clinical-grade method of expansion coupled with SB-mediated gene transfer, in the absence of any process of selection, has the chance to improve the range of applications of adoptive cell therapy protocol to control early relapse in high-risk leukemic patients.

Disclosure of Interest: None declared. 


\section{WP16}

Caspase 9 transduced T-cells after haploidentical stem cell transplantation

F. Locatelli $i^{1, *}$

${ }^{1}$ Ospedale Bambino Gesù, University of Pavia, Rome, Italy

Abstract: We have recently developed a new method of haploidentical hematopoietic stem cell transplantation (haplo-HSCT) based on the physical elimination of a $\beta+\mathrm{T}$-cells and B cells. This approach permits to leave in the graft both $\gamma \delta+$ T-cells and NK cells, this translating into a significant clinical advantage, since patients can benefit from donorderived mature NK cells already in the first weeks after the allograft. We recently completed a formally registered study (NCT01810120), started in November 2010, recruiting 80 children with acute leukemia (AL) and 33 patients with nonmalignant disorders. Despite remarkable results in terms of both relapse incidence and transplant-related mortality (largely below 10\%), recovery of adaptive immunity is still sub-optimal and requires improvement. The add-back of donor T cells expressing a suicide gene is a promising strategy to further improve immune-reconstitution after haplo-HSCT. A chimeric gene incorporating the death domain of inducible caspase 9 (iC9) has been used in a phase $\mathrm{I} / \mathrm{Il}$ clinical trial conducted in USA (Di Stasi et al. N Engl J Med 2011, Zhou et al. Blood 2014). iCasp9-modified T-cells can contribute to T-cell immune-reconstitution after T cell-depleted haplo-HSCT and could rapidly be eliminated by the administration of a dimerizing molecule, AP1903, if acute graft-versus-host disease (GvHD) occurs, with disappearance of disease signs/ symptoms. With the aim of further improving the immunereconstitution in haplo-HSCT, after having obtained the approval by the regulatory agencies, we started in November 2014 a phase I/II study enrolling children with either malignant or non-malignant disorders who receive TCR-aß/B-cell depleted haplo-HSCT, followed by the infusion of titrated numbers of iCasp9 T-cells on day $14 \pm 4$ (registration study number NCT02065869). The clinical study design consisted of a phase I portion, aimed at determining the maximum tolerated and/or recommended dose (MTD/RD) of iCasp9modified T-cells, in which patients are infused, in a classical 3 +3 design, with 3 different escalating doses $\left(2.5 \times 10^{5}, 5 \times 10^{5}\right.$ and $1 \times 10^{6}$ cells/kg recipient ideal body weight). This portion of the study has been completed and $1 \times 10^{6}$ cells iCasp9modified T-cells/kg was the RD for the phase II portion of the study. Overall, considering both the phase I and II portions of the study, 40 patients have already been enrolled in the trial and, remarkably, none of them died due to transplant-related complications. In all patients, iCasp9-modified T-cells engrafted, expanded and persisted over time, being still detectable even in patients with a follow-up longer than 1 year. These cells contribute to accelerate the recovery of adaptive immunity, with improved clinical outcome. A more detailed analysis of the impact of iCasp9-modified T-cells on both patient's outcomes and immune recovery will be presented during the meeting.

Disclosure of Interest: None declared.

\section{WP20}

Refining the role of high-dose chemotherapy and new promise of immunotherapy for advanced and relapsed germ cell tumors

A. Necchi ${ }^{1, *}$ on behalf of EBMT Solid Tumors Working Party ${ }^{1}$ Medical Oncology, FONDAZIONE IRCCS ISTITUTO NAZIONALE DEI TUMORI, Milano, Italy

Abstract: Since the late Nineties the intensification of the dose of chemotherapy with bone marrow or hematopoietic stem cell support held promise for patients with advanced germ cell tumors (GCTs) once primary chemotherapy has failed. High-dose chemotherapy (HDCT) has nowadays a recognized indication in the salvage setting of advanced GCTs and is steadily utilized worldwide.
In the first-salvage setting the only prospective randomized trial comparing conventional-dose chemotherapy (CDCT) to HDCT failed to show a PFS/OS benefit. For this reason, an international phase 3 study comparing CDCT (i.e. 4 cycles of paclitaxel, ifosfamide, and cisplatin) and HDCT (2 cycles of induction paclitaxel and ifosfamide, followed by 3 cycles of high-dose carboplatin and etoposide [TI-CE]) is now open to accrual in Europe (under the leadership of EORTC) and United States (TIGER trial).

Yet there are still other pending issues, mainly concerning the selection of patients who are more suitable for HDCT, the optimal mobilization strategy, and the inclusion of HDCT in the overall strategy of difficult-to-treat GCT.

Recently the EBMT-STWP has conducted a retrospective study on the outcomes of HDCT administered in the last 10 years in Europe. Of note, progression to induction $\mathrm{CT}$ resulted to be the most negatively influential factor on both PFS and OS, while prior paclitaxel therapy did not (Necchi et al, Bone Marrow Transplant 2015). These results did reflect what is currently perceived across the European centers and they yield the potential to refine the outcomes of HDCT in clinical trials by better selecting patients.

The few patients who fail to be cured following salvage HDCT have an extremely poor prognosis and long-term remissions have been documented in less than $10 \%$ of cases.

Hence new promising targets are awaited in this disease, yet immunotherapy is at the very early stage in GCT. CD30 is being targeted in an ongoing phase 2 study with the antibody-drug conjugate brentuximab vedotin (BV) and early results are promising. Most importantly, substantial changes in tumor microenvironment are suggested after treatment with BV, leading to unmask a number of other druggable targets (NCT01851200, Necchi et al, Genitourinary Cancers Symposium 2016). Programmed death ligand-1 (PD-L1) expression has been documented in the majority of cases of both seminoma (73\%) and nonseminoma (64\%) from a large retrospective study on 479 tumor samples (Fankhauser et al, Br J Cancer 2015). Moreover, PDL-1 expression alone from archived tissue, measured by immunohistochemistry (IHC) may not be predictive of response to PD-1/PDL-1 therapy. Dynamic changes of immunological markers may be crucial in understanding the mechanisms of drug sensitivity. Clinical trials with anti-PD1/PD-L1 antibodies are being started in Europe and US in the salvage setting.

These developments in therapeutics would lead to the design of potentially effective sequential strategies of HDCT and immunotherapy with the aim to improve survival.

Finally, further retrospective studies are being promoted by the EBMT-STWP, primarily as regards the long-term side effects of HDCT and the incidence of second-cancers.

Disclosure of Interest: None declared.

\section{WP21}

Prospective Studies of High-Dose chemotherapy with PBPC-Support in Breast Cancer

S. Rodenhuis, ${ }^{1, *}$

${ }^{1}$ Medical Oncology, NETHERLANDS CANCER INSTITUTE, Amsterdam, Netherlands

\section{Abstract: Retrospective and Prospective Studies in Breast Cancer}

In 1993, Peters published the results of a phase II study of high-dose chemotherapy. It showed a $70 \%$ relapse-free survival rate at 3 years for patients with high-risk breast cancer (HRBC), which was clearly better than that (40\%) in matched controls who had received second-generation adjuvant chemotherapy. In the following years, breast cancer became the primary indication for autologous transplants world-wide. In the US, health care insurance companies reluctantly reimbursed this treatment strategy as randomized evidence for its efficacy had been reported by Bezwoda in 1995. When the Bezwoda study was shown to represent an extreme case of scientific fraud in 2000 , and the results from 
controlled studies failed to support the results of Peters, funding for high-dose therapy ceased and any remaining interest in high-dose therapy for breast cancer became politically incorrect.

At that time, a total of 15 controlled studies had been performed internationally in the adjuvant setting, and six in metastatic patients. Meta-analyses by Berry et al in 2011 concluded that there was a significant RFS benefit in the HRBC setting, which did not translate into an OS advantage. If the analysis was limited to the 10 studies with a 'symmetrical design', however, the OS was significantly better for the highdose group $(P=0.031)$. Subgroup analyses were consistent with a substantial benefit for patients with triple-negative disease. Other reviews of roughly the same data were slightly more positive (Wang 2012; Pedrazzoli 2015). In addition, data from the Italian National Registry (Martino 2013; Pedrazzoli 2014) have shown that high-dose therapy is safe (TRM $<1 \%$ ) rather than associated with the very high mortality rates in the early American studies.

After the year 2000, very few prospective studies of high-dose chemotherapy in breast cancer have been undertaken. In the USA, at least six studies were added to the trial registry ClinicalTrials.gov after 2000. But three of these had already been activated before 2001. A single phase II study with 41 patients from City of Hope apparently ran between 2005 and October 2014, but no results have been published yet. There appears to be some trial activity in China, as well ( 2 studies in the registry).

In Italy, a randomized phase II neo-adjuvant study of standard versus high dose chemotherapy has recently been initiated. It focusses on triple-negative tumors and it compares a standard $3^{\text {rd }}$ generation regimen ( $4 x$ epirubicin/cyclophosphamide $-4 x$ Docetaxel) with $4 x$ epirubicin/docetaxel followed by high-dose therapy with epirubicin, cyclophosphamide and thiotepa.

In the Netherlands, a small multicentre phase III study of neoadjuvant high-dose chemotherapy has recently completed patient accrual. It targets stage II or III triple-negative tumours with evidence of a homologous recombination defect (HRD). Such tumours may be hypersensitive to DNA crosslinking agents. Approximately 120 patients with HRD were randomized to receive either 6 courses of conventional chemotherapy or 4 conventional courses followed by tandem intensive therapy consisting of cyclophosphamide, thiotepa and carboplatin. This study will be followed in 2016 by an international controlled trial in the (neo) adjuvant setting, the SUBITO trial, which will employ the same methods. A single-institution Dutch study, employing the same regimens, investigates the efficacy in oligometastatic breast cancers with evidence of HRD.

The Dutch and Italian controlled studies in triple-negative disease should either confirm or refute the hypothesis that high-dose chemotherapy with PBPC-support could improve the outlook for patients with triple-negative HRBC. If positive, high-dose therapy with modern agents and modern supportive care should be re-evaluated in other breast cancer subtypes as well.

Disclosure of Interest: None declared.

\section{WP23}

LWP Keynote Debate: Mantle cell lymphoma

1st-line treatment: is autoSCT still standard? No

T. Robak ${ }^{1, *}$ on behalf of Lymphoma

${ }^{1}$ Hematology, Medical University of Lodz, Lodz, Poland

Abstract: Mantle cell lymphoma (MCL) is a heterogeneous disease with a median age at diagnosis of between 60 and 70 years. In younger patients, the introduction of more aggressive induction chemotherapy with high-dose cytarabine and rituximab, with or without autologous stem cell transplantation (ASCT), significantly improves the prognosis. However, it is not clear whether consolidation therapy with ASCT is necessary in all younger patients with newly diagnosed MCL. The long term follow-up results of a phase II study in patients treated with rituximab, hyper-fractionated cyclophosphamide, vincristine, doxorubicin and dexamethasone alternating with methotrexate and cytarabine (R-HCVAD/ $M A)$, without ASCT, has been recently reported. ${ }^{1}$ R-HCVAD/MA showed sustained efficacy, with a median overall survival (OS) exceeding 10 years. For the patients aged $\leq 65$ years who are usually candidates for ASCT consolidation, the median failure free survival (FFS) was 6.5 years. In this subgroup of patients, the median OS was 13.4 years, and failure free survival (FFS) plateaued after 10 years. In addition, freedom from disease recurrence of nearly 15 years in almost $30 \%$ of the younger patients ( $\leq 65$ years) was observed. These results seem to be similar for those obtained in patients managed with ASCT in a similar patient population. However, these excellent results have not been confirmed in multicenter studies or compared with ASCT-based therapy in phase III trials. In addition, new targeted agents, i.e. bortezomib, lenalidomide and ibrutinib, can be incorporated into frontline therapy combinations and maintenance strategies in younger patients in the near future. The results of recent trials show that non-transplant therapy with these new drugs can lead to prolonged remissions in previously untreated and relapsed/refractory patients. ${ }^{2,3}$ These therapies are highly effective and less toxic than standard high-dose chemotherapy with ASCT consolidation. Bortezomib is the first proteasome inhibitor which has been approved recently for first-line treatment, both in the US and the EU. The approval was based on the results of a phase III clinical trial comparing VR-CAP (bortezomib plus rituximab, cyclophosphamide, doxorubicin and prednisone) with the R-CHOP regimen. ${ }^{2}$ Bortezomib, when used in the VR-CAP regimen, has demonstrated improved outcomes for newly-diagnosed patients with $M C L, 65$ years old and older. Another very effective first-line therapy in $M C L$ is that of lenalidomide combined with rituximab. ${ }^{3}$ In a group of patients with a median age of 65 years, the overall response rate was $92 \%$ and the complete response rate was $64 \%$. Two-year progressionfree survival was $85 \%$ and two-year overall survival $97 \%$. Several trials have demonstrated that patients with a high MCL International Prognostic Index (MIPI) score were more likely to progress and die after ASCT consolidation compared with low-intermediate risk patients. For those patients, novel therapeutic strategies based on targeted drugs are urgently needed. In addition, patients achieving MRD-negative remissions after induction with alternating R-CHOP and R-DHAP therapy achieve significantly longer PFS and OS than patients with an MRD-positive status. For those MRD negative patients, ASCT consolidation is probably not necessary. Finally, a maintenance approach based on rituximab or other targeted drugs has been found to offer effective management in older patients. It is possible that novel therapies and maintenance strategies can replace ASCT in younger population of patients. References

1. Chihara D, Cheah CY, Westin JR, et al. Brit J Haematol., 2016;172:80-8.

2. Robak T, Huang H, Jin J, et al. N Engl J Med 2015;372:944-53. 3. Ruan J, Martin P, Shah B, et al. N Engl J Med. 2015;373: 1835-44.

Disclosure of Interest: None declared.

\section{WP28}

Optimal induction, consolidation and maintenance before and after autologous stem cell transplantation in 2016 J. Blade ${ }^{1, *}$

${ }^{1}$ Amiloidosis and Myeloma Unit. IDIBAPS Hospital Clinic, Barcelona, Spain

Abstract: Induction therapy followed by high-dose melphalan/autologous stem-cell support (ASCT) is the standard of care in younger patients with multiple myeloma (MM). A bortezomib-based three-drug containing regimen (VTD, VCD, PAD or VRD) has resulted in high complete remission (CR) rates, up to $35 \%$ and $46 \%$ in an intention-to- treat basis, preand post-transplant, respectively. It seems that bortezomib 
and IMiDs have a synergistic effect and in consequence this combination would be preferable. There are ongoing trials exploring the potential benefit of adding a MoAb (i.e., the antiCD38 daratumumab) to the "classic" triple regimens. The number of induction cycles ranges between 3 and 6 . The author of the present abstract prefers 6 cycles when using bortezomib in combination with IMiDs in order to maximize its potential synergy. Patients with high-risk cytogenetics have an inferior outcome even when treated with novel agents. In this subset, tandem ASCT seems to be of benefit. The concept of post-transplant consolidation consists of the administration of additional chemotherapy cycles, usually the ones used during the induction period, at full doses over a short period of time, usually between 2 and 4 months in order to further decrease the post-transplant tumour burden, including the evaluation of the minimal residual disease before and after consolidation. This promising approach is being increasingly explored in ongoing trials and will likely be established in clinical practice. The maintenance therapy consists of the administration of low-dose drugs over a long term periods ranging from 1 to 3 years or in some instances indefinitely until undue toxicity or disease progression. The aim of maintenance is to maintain a low-tumour burden and delay relapse. The best results have been reported with IMiDs, particularly with lenalidomide, although the issues of more resistant disease at relapse and the second primary malignancies have raised some concerns. The potential synergy between lenalidomide and proteasome inhibitors (i.e., ixazomib), as well as the efficacy of MoAbs, are being explored in current trials. Finally, the main goal of ASCT in MM is the achievement of a prolonged PFS and to try to increase the cure fraction (operational cure) and the will only be reached by a "Refined Total Therapy" approach including the best induction, consolidation and maintenance, incorporating the new available drugs.

Disclosure of Interest: None declared.

\section{WP29}

Myeloma Salvage therapy options in $\mathbf{2 0 1 6}$

L. Garderet ${ }^{1, *}$ on behalf of Chronic Malignancies Working Party ${ }^{1}$ Hopital Saint Antoine, Paris, France

Abstract: Induction regimens incorporating thalidomide, lenalidomide, and/or bortezomib are now standard for newly diagnosed multiple myeloma (MM). Eligible patients may undergo high dose therapy with autologous stem cell transplantation (ASCT), which deepens and prolongs the therapeutic response and improves outcome, especially when integrated with novel agents. Consolidation/maintenance post ASCT not only upgrades but also deepens the response which translates into improved progression-free survival. Current approaches to management of newly diagnosed MM have significantly increased survival in MM. However, even patients who achieve a high quality and prolonged duration of response with initial therapy will ultimately relapse.

In undertaking the relapse diagnostic evaluation, it is important to consider various factors that will influence treatment strategy. These include characteristics of the disease itself, response and duration of response to prior therapy including treatment related toxicities, eligibility for stem cell transplantation, and characteristics of the individual patient. Patients are usually retreated with a two or three drugs combination which can include an immune modulatory drug (IMiD), a proteasome inhibitor, steroids and an alkylating agent. Monoclonal antibodies will soon be added to these combinations. Once in a state of new remission, one may consider transplantation and if not continuous treatment until the next progression. For patients transplant eligible, in contrast to the upfront setting in which the role of ASCT as consolidation of a 1st remission is well established, the role of high dose therapy with autologous or allogeneic stem cell transplantation has not been extensively studied in MM patients relapsing after primary therapy. Experts made recently the following consensus statements:
For salvage ASCT: 1. In transplant eligible patients relapsing after primary therapy that did not include an ASCT, ASCT as part of salvage therapy should be considered standard; 2. ASCT should be considered appropriate therapy for any patients relapsing after primary therapy that includes an ASCT with initial remission duration of more than 18 months; 3. ASCT can be used as a bridging strategy to allogeneic transplantation; 4 . The role of post salvage ASCT maintenance needs to be explored in the context of well designed prospective trials that should include new agents such as monoclonal antibodies, IMiD and oral proteasome inhibitors; 5. ASCT consolidation should be explored as a strategy to develop novel conditioning regimens or post ASCT strategies in patients with short (less than 18 months remissions) after primary therapy; 6 . Prospective randomized trials need to be performed to define the role of salvage ASCT in patients relapsing after primary therapy comparing to "best non ASCT" therapy. The expert committee also underscored the importance of collecting enough hematopoietic stem cells to perform two transplants early in the course of the disease. In regards to allogeneic hematopoietic stem cell transplantation (HCT), the expert committee agreed on the following consensus statements: 1. Allogeneic HCT should be considered appropriate therapy for any eligible patient with early relapse (less than 24 months) after primary therapy that included an ASCT and/or high risk features (i.e cytogenetics, extramedullary disease, plasma cell leukemia or high LDH); 2. Allogeneic HCT should be performed in the context of a clinical trial if possible; 3 . The role of post allogeneic HCT maintenance therapy needs to be explored in the context of well designed prospective trials; 4 . Prospective randomized trials need to be performed to define the role salvage allogeneic HCT in patients with MM relapsing after primary therapy.

References. Giralt S, Garderet L, Durie B, et al. BBMT 2015 Dec;21(12):2039-51. Laubach J, Garderet L, Mahindra A, et al. Leukemia 2015 in press.

Disclosure of Interest: None declared.

\section{WP30}

Novel agents before and after Stem Cell Transplantation for Myeloma beyond 2016

E. M. Ocio ${ }^{1, *}$, M.-V. Mateos ${ }^{1}$

${ }^{1}$ Hematology, University Hospital of Salamanca-IBSAL, IBMCC (USAL-CSIC), Salamanca, Spain

Abstract: Treatment of MM was based for many decades, in the use of chemotherapeutic agents, mainly alkylators; however, the appearance of agents with novel mechanisms of action resulted in a therapeutic revolution, and proteasome inhibitors such as bortezomib, and immunomodulatory agents such as thalidomide and lenalidomide resulted in a marked improvement in the survival of both young and elderly MM patients. Subsequent investigation has led to the development of second and third generations of agents belonging to those families, and, what is probably more important, of drugs with novel mechanisms of action that have demonstrated to be also effective in this disease. In fact, after the already mentioned first generation of novel agents, six new drugs have been approved for the treatment of relapsed MM patients: pomalidomide in combination with dexamethasone; two novel proteasome inhibitors: carfilzomib (alone and in combination with lenalidomide and dexamethasone), and ixazomib (in combination with lenalidomide and dexamethasone); the DACi panobinostat in combination with bortezomib and dexamethasone; and finally two different monoclonal antibodies (MoAb), elotuzumab (anti SLMF-7) in combination with lenalidomide and dexamethasone, and daratumumab (anti-CD38) with quite impressive results as a single agent. Moreover, other agents are in clinical evaluation with promising results. This is the case of agents activating the immune response against myeloma (anti-PD1/PD-L1 MoAb) or small molecules that target different pro-survival proteins (PIM-kinases, XPO-1, KSP or MEK). 
However, even in these novel agents' era, high dose melphalan followed by stem cell support is still considered as a backbone in the treatment of transplant-candidate MM patients. As has been said, most of the recently developed novel agents have been approved for the treatment of MM patients at relapse, but they are already being used in the peri-trasplant situation in order to improve the results of this procedure. In this regards some trials have substituted bortezomib for carfilzomib in induction regimes before transplant, and it has been combined with lenalidomide +dexamethasone or with thalidomide+dexamethasone with or without cyclophosphamide, with a VGPR rate or better before transplant superior to $50 \%$. Moreover, some of these trials included a consolidation phase after transplant with the same combination what clearly improved the responses. In a more optimistic approach, the Spanish MM group is currently enrolling patients in the "CESAR" trial in which transplanteligible smoldering $\mathrm{MM}$ patients with high risk of progression are being treated with KRD (Carfilzomib+Lenalidomide+dexamethasone) as induction followed by ASCT, a consolidation with KRD and a maintenance phase, with the objective of achieving the cure of some of these patients. Probably a next step would be to incorporate MoAb to this cocktail of agents. Finally, some of these novel agents, are also being used in the maintenance setting. The main representative is ixazomib, a second generation proteasome inhibitor that on top of its excellent tolerability is of oral administration. As an example, this agent is being tested alone or in combination with lenalidomide and dexamethasone in some placebo-controlled trials.

In summary, the recent approval of novel agents and mechanisms of action has increased treatment options for multiple myeloma patients. Although these innovations, are nowadays mainly focused on the relapsed situation, they are already being explored in the first line setting, and it is reasonable to think that in the near future very effective combinations including many of these agents as induction, consolidation and maintenance will be incorporated in the clinical practice.

Disclosure of Interest: E. Ocio Funding from: Amgen/Onyx Celgene, Novartis, BMS, Janssen, Takeda, Pharmamar, Array Pharmaceuticals, Mundipharma, M.-V. Mateos Funding from: Amgen/Onyx, Celgene, Novartis, BMS, Janssen, Takeda, Pharmamar.

\section{WP40}

Outcomes of allogeneic stem cell transplantation for acquired pure red cell aplasia. An ongoing analysis of the EBMT Severe Aplastic Anemia working party

C. Halkes ${ }^{1, *}$, L. de Wreede ${ }^{2}$, J. Knol-Bout ${ }^{3}$, A. Kulagin ${ }^{4}$, A. Risitano ${ }^{5}$, J. Marsh ${ }^{6}$, R. Peffault de la Tour ${ }^{7}$, J. Passweg ${ }^{8}$, C. Dufour ${ }^{9}$ on behalf of EBMT Severe Aplastic Anemia Working Group

${ }^{1}$ Hematology, Leiden University Medical Centre, ${ }^{2}$ Medical Statistics, Leiden University Medical Centre, ${ }^{3}$ EBMT Data office, Leiden, Netherlands, ${ }^{4}$ Saint Petersburg State Medical Pavlov University, St. Petersburg, Russian Federation, ${ }^{5}$ University of Napoli, Naples, Italy, ${ }^{6}$ King's Denmark Hill, London, United Kingdom, ${ }^{7}$ Hopital St. Louis, Paris, France, ${ }^{8}$ University Hospital Basel, Basel, Switzerland, ${ }^{9}$ Istituto Giannina Gaslini, Genova, Italy

Introduction: Acquired Pure Red Cell Aplasia (PRCA) is a rare haematological disease characterized by severe anaemia associated with reticulocytopenia and absence of erythroblasts in an otherwise normal bone marrow. It may present as a primary haematological disorder or secondary to parvovirus infection, collagen disease, leukemia, lymphoma, thymoma, solid tumours, treatment with recombinant human erythropoietin or other drugs. Treatment of primary PRCA or secondary PRCA which does not respond to treatment of the associated disease, is based on therapy with immunosuppressive drugs like corticosteroids, cyclosporine, cyclophosphamide, anti thymocyte globulin (ATG) or alemtuzumab. If PRCA persists despite these treatment modalities, treatment can be supportive (transfusions of red blood cells and iron chelation) or a HSCT could be offered. Case reports describe the potential benefit of treatment with an allogeneic HSCT but no cohort studies were published about PRCA patients receiving an allogeneic HSCT. In order to determine the efficacy and safety of HSCT in the treatment of patients with acquired PRCA, we collect data on transplant outcomes in patients who underwent an allogeneic HSCT for acquired PRCA.

Material (or patients) and methods: All patients from the EBMT database will be selected who underwent a first allogeneic HSCT for acquired PRCA between 2000 and 2013 The following data are collected: baseline patient characteristics (gender, age, number of transfusions before transplantation), disease (date of diagnosis, primary or secondary PRCA, previous treatment), transplantation protocol (stem cell source, graft manipulation, donor characteristics, conditioning regimen, GVHD prophylaxis) and post transplantation events (engraftment, acute GVHD, chronic GVHD, transfusions of red blood cells, need for second transplantation last date of follow-up, cause of death). Transplantation centres are contacted in order to collect the missing data. Primary outcome of this analysis will be disease-free survival up to 1 and 5 years after HSCT, which is defined as alive with engraftment and no transfusions for the treatment of PRCA and no second transplantation. Secondary Outcomes will be overall survival, incidence of acute and chronic GVHD and engraftment at 1 and 5 years.

Results: From the EBMT database 68 patients (50\% males) who underwent an allogeneic HSCT for acquired PRCA were identified. Median age at time of HSCT was 20 years (range 2 to 66). Bone marrow was used as stem cell source in 33 patients (49\%) and donors were HLA identical siblings in 34 (50\%). Transplantation Centres are being contacted to provide more detailed information on disease status prior to transplant and follow up of patients.

Conclusion: Acquired PRCA is a rare indication for allogeneic HSCT. Analysis to the efficacy and safety of this procedure is being done by the EBMT Working Party on Severe Aplastic Anemia.

Disclosure of Interest: None declared.

\section{WP41}

HCT outcome in adult patients with Fanconi anemia

M. Bierings ${ }^{1, *}$ on behalf of Severe Aplastic Anemia, C. Bonfim ${ }^{2}$, M. Aljurf ${ }^{3}$, P. Mehta ${ }^{4}$, R. Peffault de la Tour ${ }^{5}$, C. Knol ${ }^{6}$, G. Sucak', T. B. Othman ${ }^{8}$, G. Socie, K. Thomson ${ }^{9}$, G. McQuaker $^{10}$

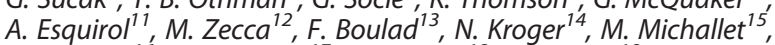
A. Risitano ${ }^{16}, G$. Ehninger ${ }^{17}$, R. Porcher ${ }^{18}$, C. Dufour ${ }^{19}$

${ }^{7}$ Hematology/Stem cell transplant, Utrecht University Children's Hospital, Utrecht, Netherlands, ${ }^{2}$ Federal University of Parana, Curitiba, Brazil, ${ }^{3}$ Hematology, King Faisal Specialist Hospital \& Research Centre, Riyadh, Saudi Arabia, ${ }^{4}$ Division of Bone Marrow Transplant and Immune Deficiency, Cincinnati Children's Hospital Medical Centre, Cincinnati, United States, ${ }^{5}$ Hematology, Hopital St Louis, Paris, France, ${ }^{6} E B M T$ Data Office, EBMT, Leiden, Netherlands, 7 Gazi Universitesi, Ankara, Turkey, ${ }^{8}$ Centre National de Greffe de Moelle, Tunis, Tunisia, ${ }^{9}$ University College London Hospital, London, ${ }^{10}$ Beatson West of Scotland Cancer Centre, Glasgow, United Kingdom, "Hospital Santa Creu i Sant Pau, Barcelona, Spain, ${ }^{12}$ Pediatric Hematology/Oncology, Fondazione IRCCS Policlinico San Matteo, Pavia, Italy, ${ }^{13}$ Pediatric Bone Marrow Transplant Service, Memorial Sloan Kettering, New York, United States, ${ }^{14}$ University Hospital Eppendorf, Hamburg, Germany, ${ }^{15}$ Centre Hospitalier Lyon Sud, Lyon, France, ${ }^{16}$ University of Napoli, Naples, Italy, ${ }^{17}$ Universitatsklinikum Dresden, Dresden, Germany, ${ }^{18}$ University Paris Descartes, Paris, France, "Istituto Giannina Gaslini, Genova, Italy

\section{Abstract: HCT Outcome in adult patients with Fanconi} Anemia

Patients with Fanconi Anemia (FA) generally develop progressive bone marrow failure in the first decades of life and are at increased risk of developing myelodysplastic 
syndrome (MDS)/leukemia. Some patients present at adult age. Hematopoietic stem cell transplantation (HCT) is the only curative therapy. Using adapted conditioning regimens (due to increased chemo- and radiotherapy sensitivity) and adding fludarabin has resulted in greatly improved results of HCT for FA. In a recent EBMT analysis by Peffault de Latour et al (Ref1) age at transplant was a risk factor for outcome. Here we report results of our retrospective analyses of HCT outcomes in a large cohort of adult patients with FA.

All patients with FA over 18 yrs of age at HCT were included in this retrospective study. Participating centres include EBMT centers as well as other centers. Patients were transplanted between 1991 and 2014. Data on disease and transplant characteristics and transplant results were obtained.

180 patients were included in this analysis.

Patients were 18-44 yrs of age at transpant (median age 23).148 patients (82\%) were below 30 years of age at transplant. Time from diagnosis to transplant ranged from 1-13 years (median 6). 25 of 77 evaluable patients (32\%) had signs of clonal hematopoetic disease at transplant: 21 (27\%) MDS, $6(8 \%)$ acute leukemia. In 90 patients (50\%) donors were HLA identical siblings, in 7 patients (4\%) other identical relatives, in 10 patients $(6 \%)$ mismatched related donors were used; In 30 patients (17\%) donors were matched unrelated, 23 patients (13\%) had mismatched unrelated donors. In 113 patients (63\%) the stem cell source was marrow, in 55 patients (31\%) PBSC and cord blood was used in 11 patients (6\%). Ex vivo $\mathrm{T}$ cell depletion was employed in 11 patients (8\%). Conditioning regimens contained in 90 of 156 evaluable patients (58\%) fludarabin, in 150 patients (96\%) cyclophosphamide and in 44 patients $(25 \%)$ it contained TBI.
24 patients (15\%) received busulfan as part of the conditioning. In $55 \%$ of cases ATG was used as serotherapy. Engraftment occurred in $80 \%$ of cases. 17 patients had primary graft failure, 11 secondary, 5 patients died without engraftment. One patient was alive without engraftment and lost to follow up, data for 6 non-engrafting patients are still missing. aGvHD II-IV occurred in $23 \%$ of cases $(95 \% \mathrm{Cl} 17-30)$. cGvHD at 12 months was present in $29 \%$ (95 Cl 20-38) of patients surviving at 100 days. Non-relapse mortality at 12 months was $44 \%$ (95 Cl 36-51), increasing to $52 \%(95 \mathrm{Cl}$ 44-60) at 96 months. Causes of death were mainly infection (34\%), GvHD (29\%) and organ failure (14\%). Overall survival at 12 months was $54 \%(95 \mathrm{Cl} 47-62)$, decreasing to $37 \%(95 \mathrm{Cl}$ 30-46) at 96 months.

To our knowledge this is the largest dataset of adult patients with FA undergoing HCT. Our results confirm that outcomes for adults with FA remain considerably lower than results described for younger patients. Non-relapse mortality has significant impact on these poor results. Further analyses are ongoing to define additional risk factors e.g. disease status at HCT, transplant period and impact of stem cell source as well as conditioning regimen. These results emphasize the need to consider HCT at an early age in all patients with symptomatic FA as well as the need to identify novel adapted HCT approaches for adult patients with FA.

Reference 1 Peffault de la Tour R, Porcher R et al Allogeneic hematopoietic stem cell transplantation in Fanconi anemia: the European Group for Blood and Marrow Transplantion experince. Blood 2013122 4279-86.

Disclosure of Interest: None declared. 